\title{
CONSUMOS DE ENERGÍA EN LA INDUSTRIA DEL PLÁSTICO: REVISIÓN DE ESTUDIOS REALIZADOS
}

\author{
Energy consumption in plastic industry - review of studies developed
}

Carlos Andrés Vargas Isaza

M.Sc. Ingeniería de Procesos de transformación del plástico y del caucho. Instituto Tecnológico

Metropolitano. Medellín, Colombia. carlosvargas@itm.edu.co

Juan Carlos Posada Correa

M.Sc. Ingeniería en Automatización y control, Instituto Tecnológico Metropolitano. Medellín, Colombia.

juancposada@itm.edu.co

Leyla Yamile Jaramillo Zapata

PhD (c). Ingeniería Ciencia y Tecnología de Materiales. Instituto Tecnológico Metropolitano. Medellín, Colombia.eylajaramillo@itm.edu.co

\section{Luis Alberto García}

MBA en Administración. Instituto Tecnológico Metropolitano. Medellín, Colombia. luisgarcia@itm.edu.co

\section{Cómo citar / How to cite}

Vargas, C., Posada, J., Jaramillo, L., y García, L. (2015). Consumo de energía en la industria del plástico: revisión de estudios realizados. Revista CEA, 1(1), 93-107.

Recibido: 28 de julio de 2014

Aceptado: 9 de septiembre de 2014

\section{Resumen}

El nivel de consumo energético para la producción de artículos plásticos es una variable que en los últimos años ha cobrado gran importancia, ya que tiene no solo un impacto ambiental en su uso racional sino que tiene un costo representativo para la producción. El artículo contiene una breve justificación de la relevancia de cuantificar el consumo de energía requerido para la producción de piezas plásticas, como también la forma de medirlo en variables tales como consumos fijos y variables, así como determinar el consumo de energía específico por planta de procesamiento, proceso y por equipos. Posteriormente, se hace un recuento de diferentes estudios de consumo y uso eficiente de la energía más relevantes en la industria del plástico, a nivel regional, nacional e internacional. El presente artículo es logrado con el objetivo de proveer un material de referencia básico a investigadores interesados en este tema.

Palabras clave: consumo de energía específica, procesos de transformación de polímeros (plásticos), eficiencia energética en la industria del plástico.

\begin{abstract}
The level of energy consumption for the production of plastics is a variable that in recent years has gained great importance due to its environmental impact on energy rational use, and its representative cost for production. This paper contains a brief explanation of the importance of quantifying the energy consumption required for the production of plastic parts, as well as how to measure variables such as consumption fixed and variable, and determine the specific energy consumption for processing plants, for processes and equipment. Subsequently, it describes several studies about energy consumption rates relevant to the plastics industry in Colombia and different countries that count large polymer processing plants. This paper is made in order to provide a basic reference material to researchers interested in this topic.
\end{abstract}

Keywords: Specific Energy Consumption (sec), polymer processing, energy efficiency in plastic plants. 


\section{INTRODUCCIÓN}

El sector productivo de plásticos en Colombia ha tenido en los últimos años un crecimiento sostenido. La capacidad instalada total para la producción de resinas plásticas pasó de 1.04 millones de toneladas en 2008 a 1.15 millones en 2010, y en cuanto al procesamiento nacional de resinas, se pasó de 853 mil toneladas en 2008 a 909 mil toneladas en 2010 (Acoplásticos, 2011). A nivel internacional, la producción de plásticos ha tenido una tendencia creciente, estimada al año 2011 en 270 millones de toneladas (CIPEC \& Industry, 2007; Gough, Gaffney, \& Mccann, 2006; Padur \& MacDougall, 2011; PlasticsEurope \& Organisations, 2012; Suosa, 2014). La competitividad internacional, ha impulsado a las empresas del sector a ser cada vez más productivas, y efectivas en el cumplimiento de estándares de calidad en sus productos.

En años recientes, los consumos energéticos generados por las empresas del sector plástico han cobrado gran importancia por el incremento de costos en la energía (Kent, 2009; Santa María et al., 2009), el cumplimiento de normativas del uso racional de la energía y la implementación de sistemas de gestión energéticos o ambientales (Prias, 2010), así como los potenciales ahorros de energía que se pueden obtener (Ipsom, 2011). Son pocas las empresas de plásticos en Colombia que tienen una gestión energética aplicada a sus procesos de transformación y con un marco de referencia internacional, que permitan verificar si sus procesos tienen uso racional de la energía. Tangram Technology (2013) es un grupo de investigadores en Europa que lidera el tema de la gestión energética en la industria plástica. Este grupo ha establecido diferentes estándares de consumos de energía para diferentes líneas de producción de plásticos. En la industria de plásticos en Colombia, se tiene desconocimiento general sobre el nivel de uso racional de la energía, con respecto a estos estándares internacionales establecidos.

La eléctrica es la principal fuente de energía para el accionamiento de los equipos de procesamiento de plásticos, y puede llegar a representar entre el $4 \%$ y el $10 \%$ de los costos operacionales en una planta de transformación (Naranjo, 2012). En procesos como la extrusión para generar productos tales como películas, láminas y perfiles, la energía eléctrica se requiere para poner en funcionamiento el motor que suministra el torque necesario para dar movimiento al husillo extrusor, encargado de transportar, fundir y homogenizar el material que posteriormente será moldeado en un cabezal de extrusión, otorgando la forma final a los productos (Bernia Espert, 2011; Heur \& Verheijen, 2009; RECIPE, 2006). Adicionalmente, procesos posteriores a la extrusión, como sistemas de estirado y corte, también pueden requerir el uso de motores eléctricos. En condiciones ideales, el 50\% del total de la energía se usa para impulsar el tornillo de extrusión y el restante es empleado para sistemas de calentamiento, refrigeración, equipos auxiliares y servicios (Novoa et al., 2009).

En el proceso de inyección se produce una gama de artículos que van desde aplicaciones para el hogar, construcción, medicina, autopartes, entre otros. Al igual que en el proceso de extrusión, en la inyección se requiere el accionamiento por motor de un tornillo de inyección, para transportar, fundir e inyectar el material en un molde que da la forma final del producto. A diferencia del proceso de extrusión, en el proceso de inyección se generan mayores presiones de procesamiento, lo cual va directamente ligado al consumo de energía eléctrica. De hecho el costo energético de las máquinas inyectoras en una planta de procesamiento por inyección, puede llegar a ser hasta $60 \%$ del consumo total de la planta (Heur \& Verheijen, 2009). 
Entre los procesos descritos y los de transformación existentes tales como soplado, termoformado, rotomoldeo, entre otros, se tiene como común denominador el uso de motores, sistemas de calefacción y enfriamiento, sistemas hidráulicos y aire comprimido, donde el consumo de energía eléctrica es el mayor demandante del global de energía de los procesos (Heur \& Verheijen, 2009; McCart \& Kearns, 2012; RECIPE, 2006; Unido \& Miti, 1995).

En el presente artículo se describirá en primera instancia el concepto de eficiencia energética y algunos resultados obtenidos en el procesamiento de plásticos. Posteriormente, se describen algunos métodos para determinar consumos de energía, específicamente en empresas transformadoras de plásticos. Adicionalmente, se realizará un resumen y estado actual de diferentes estudios de índices consumos de energía, realizados en empresas del sector de plásticos a nivel nacional e internacional.

\section{EFICIENCIA ENERGÉTICA EN EL PROCESAMIENTO DE PLÁSTICOS}

La eficiencia energética se puede definir como la relación entre las energías utilizadas y el volumen o cantidad producida o movilizada (Tanaka, 2008). La producción y distribución de energía eléctrica en el mundo tiene un alto costo y no es eficiente. Se estima que solo un $35 \%$ del total de energía producida por todas las fuentes disponibles actualmente, es aprovechada por la industria y otros sectores (IEA, 2013).

La eficiencia energética se puede impactar desde el diseño y la implementación de tecnologías desarrolladas con criterios de uso eficiente y racional de la energía (Arburg, 2010; García, 2010; Ipsom, 2012; Joseph, 2003; Kent, 2008; Kraussmaffei, 2010; RECIPE, 2005, 2006; Thiriez \& Gutowski, 2006), los procedimientos operativos (Heur
\& Verheijen, 2009; Noriega \& Estrada, 2011; RECIPE, 2006) y el mantenimiento de equipos (Heur \& Verheijen, 2009; RECIPE, 2006). Huang \& Yang (2012), describen el concepto de los cuatro factores aplicados a empresas transformadoras de plástico, que implementa en forma adecuada los criterios listados de impacto en la eficiencia energética. El concepto de los cuatro factores consiste en mejorar en el doble la eficiencia vía procesamiento y tiempo de ciclo, y reducir el consumo de recursos en la mitad con menor consumo de energía, materia prima y agua.

Tanaka (2008) realiza una descripción y estudio detallado sobre la forma de medir la eficiencia energética en las industrias manufactureras, donde se definen cuatro tipos de indicadores de eficiencia energética: (1) la energía térmica del equipo que es el cálculo de la energía de salida sobre la energía de entrada; (2) el consumo de energía de cantidad absoluta medido en unidades de energía calórica; (3) tasas de difusión de eficiencia energética en plantas y equipos; y (4) la intensidad de energía que valora el consumo de energía sobre un valor físico de salida (KWh/kg). Esta última es la más empleada, ya que al ser medida con respecto a la producción física de artículos industriales tiene la ventaja de comparar desempeños energéticos entre procesos, industrias, empresas e incluso entre países. Este indicador será el referenciado en el presente artículo.

La implementación de mejoras en la eficiencia energética puede tener un gran impacto. El Departamento de Energía de los Estados Unidos, reporta para empresas del sector de plásticos retornos de inversión inmediatos y ahorros anuales hasta US\$ 240000 (Scheihing, 2006). En España, la Agencia Andaluza de la Energía, muestra un estudio completo de programas de incrementos en la eficiencia energética en la industria plástica española. Para la industria petroquímica se hace un estudio basado en 
la eficiencia de procesos y equipos complementarios a la transformación de plásticos, con impactos del ahorro de energía en sistemas de calefacción, redes de vapor, aire comprimido, motores eléctricos, sustitución de fuentes de energía por gas natural, cogeneración, mejoras en iluminación y sistemas de producción en frío (Neelis, Worrell, \& Masanet, 2008).

\section{MÉTODOS PARA VALORAR EL CONSUMO ENERGÉTICO}

\section{Consumos fijos y variables en una empresa de plásticos}

En cualquier empresa de procesamiento de plásticos es posible determinar la carga base y de proceso, a partir de la información disponible del consumo de energía de la planta y los datos del volumen de producción. Para esto se requiere registrar el volumen de producción en kilogramos, para un número de meses o semanas y registrar el respectivo consumo de energía en kWh para los mismos periodos (Campos A., 2006). Es posible entonces correlacionar en un gráfico esta información, mediante una ecuación lineal, tal como se ilustra en la Fig. 1, para una empresa de inyección de plásticos (BarcoVision, 2008).

De acuerdo con el ejemplo presentado en la Fig. 1, la información del consumo fijo implica que así no haya producción en la planta de inyección, de igual forma existe un consumo aproximado de 152423 kWh/mes, este valor representa aproximadamente un $30 \%$ del consumo energético de la empresa. Dicho valor se encuentra dentro del rango promedio esperado ( $10 \%$ al $40 \%)$ para este tipo de empresas (Kent, 2008).

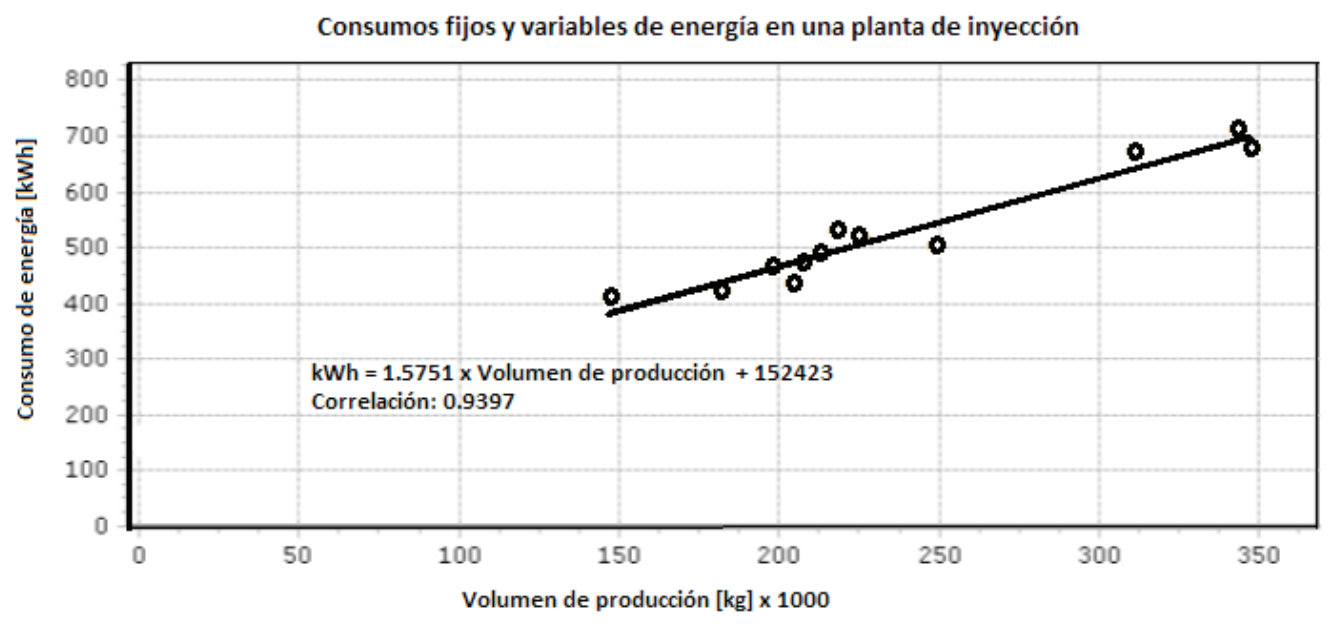

Figura 1. Consumos fijos y variables de energía en una planta de inyección (BarcoVision, 2008)

Figure 1. Fixed and variable energy consumption in an injection industrial plant (BarcoVision, 2008).

El consumo fijo se refiere generalmente a gastos asociados a máquinas y equipos de servicio en continua operación, independiente del volumen de producción de la empresa, tales como aire comprimido, sistemas de aguas de refrigeración, luminarias, equipos de transporte de materiales, entre otros. Las reducciones en el consumo fijo pueden ser realizadas en algunas ocasiones sin afectar la producción, 
calidad y operación de la planta de procesamiento de plásticos. Los ahorros en los consumos fijos son muy rentables, ya que no están directamente relacionados a la producción de la empresa.

De la Fig. 1 se puede observar también que el consumo variable es aproximadamente $1.5751 \mathrm{kWh} / \mathrm{kg}$ de plástico procesado. Este consumo muestra la eficiencia de la planta en el procesamiento de plásticos. Las reducciones en el consumo variable indican una mejora en la eficiencia de los procesos productivos. Con base en esta información es posible comparar consumos de energía históricos entre diferentes periodos de tiempo y analizar las reducciones $o$ incrementos en los consumos de energía de la empresa. Adicionalmente, a partir de la información obtenida es posible realizar pronósticos de consumos de energía con base en los consumos históricos que se comportaron por debajo del promedio, para de esta forma crear metas de reducciones de consumos energéticos (Hill \& Figueroa, 2007).

\section{Valoración energética entre plantas de procesamiento de plásticos y equipos de proceso}

Otro aspecto importante a considerar es cuantificar y comparar el consumo energético de una planta de procesamiento con respecto a otras similares. Esta valoración es posible realizarla en función del consumo específico de energía, el cual se mide en el consumo de energía ( $k W h$ ) con respecto a cantidad de material procesado (Hill \& Figueroa, 2007). Se puede hablar entonces del consumo de energía específico de una máquina de procesamiento de polímero o de una planta, una sección de la planta o de un componente de equipo, de acuerdo a la escala de producción (kilogramos) que se tenga disponible (Kent, 2008).
Si se desea estimar el consumo específico de energía de una planta de procesamiento, se debe disponer entre otras variables de la siguiente información: (1) Variables de producción tales como horas trabajadas por turnos, turnos por día, días efectivos de trabajo al año, (2) El consumo de energía global de la planta en un periodo de tiempo (Ej: un año), (3) La producción total de la planta en el mismo periodo de tiempo (Ej: un año), y (4) Número de máquinas en producción.

Para el cálculo de consumos específicos por equipo de procesamiento, se debe tener en cuenta en el momento de valorar y comparar equipos sus especificaciones técnicas $y$ tecnológicas, ya que estas tienen influencia en sus consumos energéticos. En este caso, para estimar el consumo de energía específico se requiere información más detallada de cada equipo o máquina de procesamiento a evaluar (Kent, 2008), tal como la producción promedio de la máquina de procesamiento y el consumo de energía promedio de la máquina durante su producción.

\section{CONSUMOS ENERGÉTICOS EN LA INDUSTRIA DEL PLÁSTICO}

\section{Consumos por tipo de proceso de transformación}

Con la implementación de sistemas de gestión energética, auditorías energéticas o programas de uso racional de la energía en empresas del sector de plásticos, se hace necesario cuantificar los consumos energéticos asociados a la transformación de los materiales plásticos (Kent, 2011; Noriega \& Estrada, 2011). A nivel internacional el grupo de investigadores de Tangram Technology (Kent, 2008) en Gran Bretaña, The EURECIPE report (EURECIPE, 2005) y el Department of Science Technology and Environment of Ho Chi Minh City, 
(Pethybridge, Hoi, \& Mui, 2002), han realizado varios estudios de consumos de energía por tipos de empresas de procesamiento de plásticos. Algunos valores típicos de consumo de energía específicos promedio para el proceso de inyección se encuentran entre 2.9 a $3.1 \mathrm{kWh} / \mathrm{kg}$ y para el proceso de extrusión de $1.5 \mathrm{kWh} / \mathrm{kg}$ aproximadamente, (Kent, 2008). Los procesos de transformación de plásticos mencionados corresponden a los más representativos en la actualidad.

Para llegar a este nivel de detalle en estos consumos, discriminado por tipos de proceso, se ha requerido la evaluación de los consumos específicos de energía en un grupo representativo de empresas de la región de Europa.

Para los procesos de inyección y extrusión, Tangram Technology ha generado la información de curvas de operación de consumos específicos de energía versus volúmenes de producción, en cerca de 98 empresas en todo el mundo (Kent, 2008e). En la Fig. 2 se ilustra el resumen de dicho estudio para estos dos tipos de procesamiento.

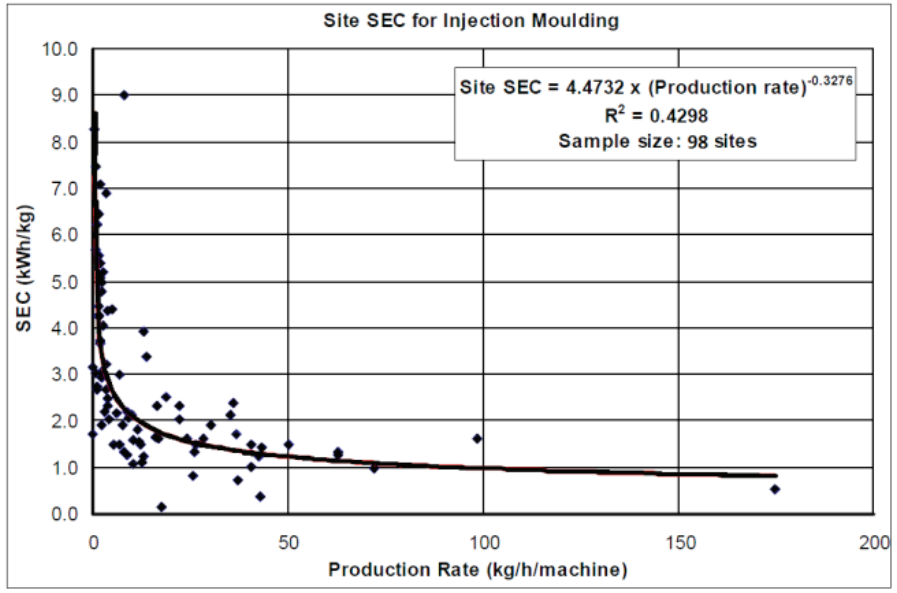

a)

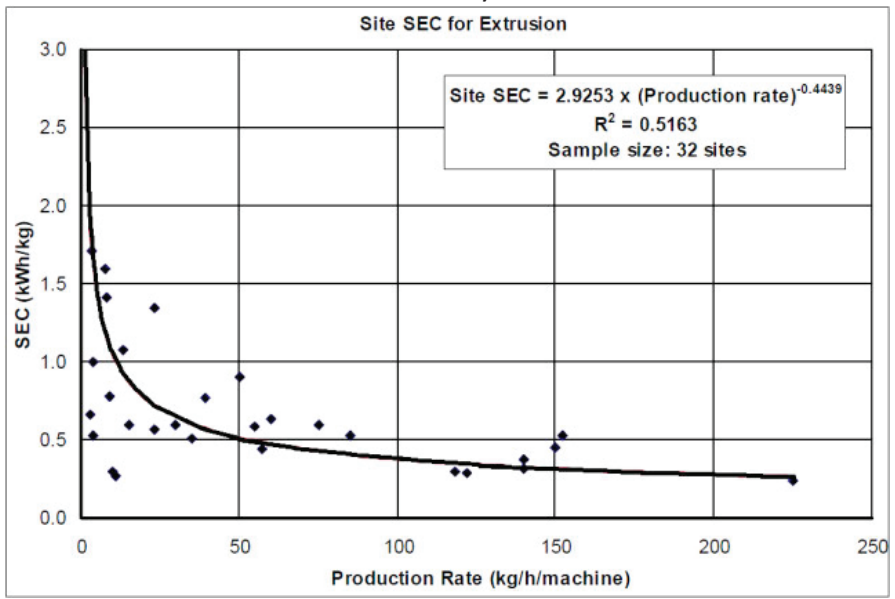

b)

Figura 2. Consumos específicos de energía de empresas de inyección (a) y extrusión (b) generados por Tangram Technology (Kent, 2008).

Figure 2. Specific energy usage in a) injection and b)extrusión industries reported by Tangram Technology (Kent, 2008). 
Para los datos obtenidos en el estudio mencionado, se deben tener en cuenta algunas condiciones como (Kent, 2008): (1) El modelo de ajuste de los consumos de energía específicos presenta un menor o mayor error de acuerdo con la cantidad de datos disponibles. (2) El uso de este modelo tiene como propósitos de encontrar valores de referencia promedios, para establecer órdenes de magnitud de valores típicos de diferentes procesos de transformación de plásticos, en este caso inyección y extrusión. (3) Se emplearon varios tamaños de máquinas de inyección y extrusión. Sin embargo, asumiendo un consumo promedio por máquina las desviaciones en el modelo no son significativas. (4) El alto grado de dispersión en la Fig. 2 se debe a las diferentes condiciones de operación de las máquinas y en consecuencia diferentes niveles de eficiencia en la producción. (5) La diferencia en los tipos de materiales procesados parece no tener influencia en el estudio global del modelo.

Se debe tener en cuenta que al ser un estudio global promedio, la alta variabilidad que se puede presentar puede absorber las variaciones en la naturaleza del material, condiciones de proceso entre otros.

Al observar los datos de la Fig. 2, se confirma lo descrito entre los consumos de los procesos de inyección y extrusión, donde los primeros corresponden en un orden de magnitud de prácticamente el doble de consumo de energía con respecto a los del proceso de extrusión. Para otros procesos de transformación de plásticos como termoformado, extrusión -soplado e inyección- soplado, entre otros, la cantidad de datos obtenidos es muy baja, solo se disponen de algunos estudios hechos por Tangram Technology, (Kent, 2008). Sin embargo, no se tiene la suficiente información para obtener conclusiones sobre la eficiencia energética de estos procesos y sus índices de consumos, lo cual puede ser una oportunidad de investigación más detallada en estos procesos de transformación.

Otro referente internacional importante fue el estudio realizado por Rapra Technology, (Colbert, 2005) para RECIPE (Reduce Energy Consumption in Plastics Engineering). Este estudio fue realizado para cerca de 165 empresas de Europa, especialmente en Alemania, España y Gran Bretaña. En los cuales se obtuvieron algunos resultados generales de los consumos de energía específicos por países y por tipo de procesos productivos de plásticos.

En otros países como Estados Unidos y Canadá, no se disponen de datos puntuales de consumos específicos de energía del sector de plásticos. Sin embargo, se tienen estudios muy completos de mejoras en la eficiencia energética en plantas de procesamiento de plásticos y cauchos, a partir de estudios de consumos de energía (KEMA, 2012; SPI \& Energy, 2005). En el gobierno de Canadá se presenta una guía completa enfocada a las industrias de procesamiento de plásticos en esta región, definiendo el perfil del sector en sus procesos y productos (CIPEC \& Industry, 2007).

\section{Consumos según tipo de maquinaria}

De igual forma, a nivel de equipos $y$ máquinas de procesamiento es posible obtener el consumo de energía específico. En este caso, se requiere la medición particular del consumo de energía promedio de la máquina durante su operación y su correspondiente producción, así como también varias mediciones, ya que en una misma máquina se pueden configurar diferentes tipos de productos (piezas de pared delgada o gruesa) y diferentes materiales y condiciones de proceso; es por este motivo que se debe hablar de un promedio de consumo de energía específica. 
La empresa Tangram Technology, ha realizado una labor investigativa, midiendo, recopilando y procesando la información de consumos de energía específica de equipos de inyección y extrusión alrededor del mundo (ver Fig. 3). Para tener en cuenta la referencia de estos datos se deben tener en cuenta la siguientes consideraciones (Kent, 2008) : (1) El estudio comparativo del consumo de energía específica (SEC) de las máquinas de procesamiento es un valor promedio y algunas combinaciones máquina/molde tienen un SEC considerablemente bajo, dependiendo de la productividad obtenida (kilogramos/hora). (2) El modelo de estudio no tiene en cuenta los diferentes tamaños de máquinas empleadas en las diferentes plantas de procesamiento. (3) Las máquinas de inyección monitoreadas fueron todas máquinas convencionales hidráulicas. (4) El alto grado de dispersión en la gráfica se debe a las diferentes condiciones de operación de las máquinas. (5) La diferencia en los tipos de materiales procesados parece no tener influencia en el estudio global del modelo.

Se debe tener en cuenta que al ser un estudio global de máquinas, la alta variabilidad que se puede presentar puede absorber las variaciones en la naturaleza del material, condiciones de proceso entre otros. Como se describieron, los anteriores estudios corresponden a consumos promedios por procesos y por máquinas (inyectoras y extrusoras). Recientemente se han venido realizando estudios más especializados por procesos, que involucran las variables de máquina, material y producción. Weissman, Gupta, Ananthanarayanan, \& Sriram (2010) proponen una metodología para estimar el consumo de energía de piezas inyectadas desde la etapa de diseño, teniendo en cuenta la información de la geometría del producto, las especificaciones técnicas de la inyectora, las propiedades del material plástico y los tiempos de entrega de producción.

LLu, Gong, Yang, \& Lu (2012) implementaron un diseño de experimentos basado en el método de Taguchi, combinado con un análisis de proceso (ANOVA) y redes neuronales para describir el modelo de optimización de inyección que incluye el consumo de energía en su análisis. Villegas \& Naranjo (2013) presentan también una metodología para mejorar la eficiencia energética en el proceso de inyección, partiendo del estado actual del procesamiento, midiendo el consumo de energía con un sistema de captación de datos, acompañado de un software que permite obtener los consumos de energía en cada etapa del ciclo de inyección, para de esta forma determinar qué etapa del proceso se puede optimizar. En este mismo sentido, Gong, Lu, Lu, \& Yang (2012) proponen un modelo de consumo de energía transición, en el cual analizando varias piezas de inyectadas logran un balance entre bajo consumo de energía y buena calidad de la pieza inyectada.

En el proceso de extrusión también se tienen algunos estudios más detallados de consumos de energía. Mena \& Estrada (2013) muestran un estudio de consumos de energía para una extrusora mono husillo con alimentación forzada en una ventana amplia de operación (15 puntos), los autores concluyen que este equipo se debe operar con altos niveles de velocidad del husillo y altas restricciones del equipo (cabezales), alcanzando una mayor estabilidad en el flujo másico, mayor eficiencia energética y menor consumo de energía específica. Cantor (2010) propone un análisis de consumo de energía para un polímero semicristalino y amorfo con tres diseños diferentes de husillos, recomendando la implementación de elementos de mezcla, un buen aislamiento de la zona de calefacción y altas velocidades de rotación con el propósito de optimizar el consumo de energía de la 
extrusora. En las zonas de calefacción Christiano, Wheeler, Baird, \& Standard (2011) proponen un nuevo diseño, el cual reduce la energía requerida para calentar el cilindro entre 24 y $33 \%$ con respecto al diseño convencional. Para extrusoras doble husillo, Canedo \& Dreiblatt (2012) desarrollaron un modelo computacional en una dimensión para calcular el consumo de energía específica, permitiendo optimizar la eficiencia de plastificación y minimizando la temperatura de fundido.

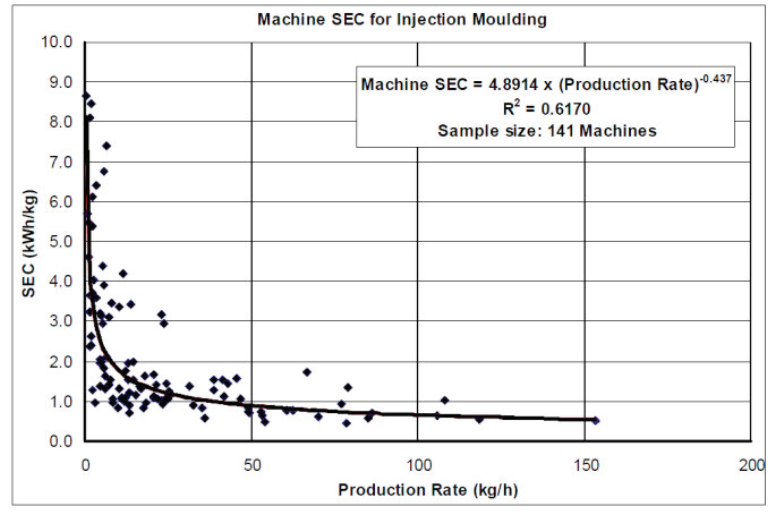

a)

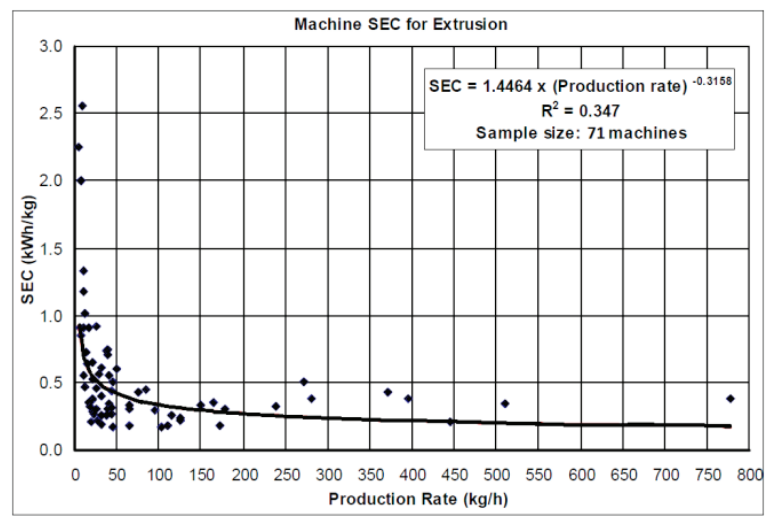

b)

Figura 3. Consumos específicos de energía en inyectoras a) y extrusoras, b) generados por Tangram Technology, (Kent, 2008).

Figure 3. Specific energy usage in a) injection moulding machines and b)extrusión machines reported by Tangram Technology, (Kent, 2008).

Como iniciativas de estudios energéticos relacionados al subsector de plásticos en Colombia, se pueden mencionar el realizado por la Cámara de Comercio de Bogotá en convenio con el Banco Interamericano de Desarrollo (BID), MIF FOMIN y la Corporación Ambiental Empresarial de Bogotá. Este estudio estuvo dirigido a definir una guía metodológica para el uso eficiente de la energía en el sector de plásticos (Novoa et al., 2009). Un primer estudio a nivel regional enfocado a determinar índices energéticos para la industria del plásticos y diferentes procesos fue el realizado por los investigadores docentes de la Universidad de los Andes René, Larrotta, \& Rey (2000), donde se realizaron mediciones para diferentes procesos de 
transformación de plásticos en empresas de la ciudad de Bogotá.

En el Instituto de Capacitación e Investigación del plástico y del Caucho, ICIPC, se han desarrollado diversos proyectos y estudios de mejoramiento energético en algunas empresas del sector plásticos en Colombia, tales como Vinipack en Bogotá, Diax y Prodenvases en el área metropolitana de Medellín (ICIPC, 2013). En donde partiendo de las mediciones energéticas actuales se pudieron pactar y lograr metas de ahorro en energía.

\section{CONCLUSIONES}

La industria del plástico es un sector de gran potencial de desarrollo a nivel nacional e internacional. Es importante tener en cuenta su impacto en los consumos energéticos requeridos, para procesar y transformar los diferentes materiales plásticos. En su mayoría, los equipos de procesamiento requieren energía eléctrica para transformar los plásticos y estos representan más de la mitad del consumo de energía global para generar un producto plástico. El consumo energético tiene una relevante influencia sobre la competitividad de las empresas de plásticos y contribuye a la mitigación del impacto ambiental de este sector manufacturero.

En lo encontrado en la literatura, se pueden distinguir dos frentes de trabajo de investigación en el consumo de energía en el sector productivo de plásticos. En primera instancia, se dispone de información de referentes internacionales de consumos de energía globales por tipos de proceso y equipos de procesamiento. Otro frente de trabajo de interés es la exploración de metodologías e investigaciones para casos de estudio más detallados de optimizaciones en consumos de energía, para procesos de transformación de plásticos (inyección y extrusión), en los cuales se tiene en cuenta el tipo de tecnología, material a procesar, geometría del producto y condiciones de producción. Para estos dos campos de trabajo se encuentra que a nivel nacional aún falta trabajo y proyectos por desarrollar en el área de la optimización del recurso energético para el procesamiento de materiales plásticos.

Normalmente, las empresas cuantifican los consumos de materia prima y la producción en kilogramos, sin embargo, no se mide la energía consumida por los procesos (kWh) y por lo tanto la relación $\mathrm{kWh} / \mathrm{kg}$ es un valor poco conocido o cuantificado, este hecho motiva la necesidad de realizar una caracterización más amplia de los consumos energéticos en las regiones de mayor desarrollo y producción del sector plástico en Colombia.

En general, los estudios realizados son iniciativas de grupos de investigación, universidades y centros de consultorías, mas no de estudios específicos de empresas. Sin embargo, en la actualidad no se tiene un estudio global energético del sector de plásticos para otras regiones importantes en la industria del plástico tales como Antioquia, Valle, Santander y la costa Atlántica (Cartagena, Barranquilla), que permita caracterizar y conocer los índices de consumo de energía de las empresas de transformación de plásticos en Colombia. Este hecho muestra una gran oportunidad para valorar la eficiencia energética de las plantas de procesamiento de plásticos de nuestras regiones y determinar potenciales acciones de mejora, para optimizar el uso racional de la energía en este sector de empresas. 


\section{REFERENCIAS}

Acoplásticos. (2011). Las materias plásticas en Colombia, principales variables de su evolución en el trienio 2008-2010. Plásticos en Colombia 2011-2012, 81-133.

Arburg (2010). Andaltec -presentación eficiencia energética Inyección rentable índice. Jornadas de eficiencia energética. Martos, España. Recuperado de: http://andaltec.org/documentaci on/pdf/1286263470.pdf

BarcoVision (2008). La monitorización de la energía acarrea un ahorro potencial enorme en las empresas del procesado de plásticos. Recuerado de: https://www.interempresas.net/Feri aVirtual/Catalogos_y_documentos/1 92311/BARCOVISION_BMS_EnergyM onitoringPlastics.pdf

Bernia, A. (2011). Técnicas para la reducción del consumo energético. Plásticos Universales, 297, 46-47.

Campos, J. C. (2006). Herramientas para el análisis de caracterización de la eficiencia energética. Recuperado de:

http://www.revistaciencias.com/ publicaciones/EpZyEkkEuulBycVx Cr.php.

Canedo, E., \& Dreiblatt, A. (2012). Distribution of specific energy in twin-screw corotating extruders using one-dimensional process simulation. ANTEC 2012.

Cantor, K. M. (2010). Analyzing extruder energy consumption. ANTEC 2010, 603-609.
Christiano, J. P., Wheeler, N., Baird, J., \& Standard, D. (2011). Improving the energy efficiency of a barrel heater - cooler design for high temperature single screw extrusion applications. ANTEC 2011, 1267-1271.

CIPEC, \& Industry, C. P. (2007). Guide to energy efficiency opportunities in the canadian plastics processing industry. Recuperado de: http://oee.nrcan.gc.ca/sites/oee. nrcan.gc.ca/files/pdf/industrial/t echnical-

info/benchmarking/plastics/docu ments/plastics-guide-englishjanuary-2008.pdf

Colbert, J. (2005). A RECIPE for energy management. Recuperado de: www.euRECIPE.com

EU RECIPE. (2005). 2005 European benchmarking survey of energy consumption and adoption of best practice. Recuperado de: http://www.ieelibrary.eu/index.php?option=com jombib\&task=showbib\&id $=583$

García, V. (2010). Ahorro Energético en los procesos de transformación en materias plásticas con el sistema Ecodry de Frigel. Jornadas de eficiencia energética, Martos, España. Recuperado de: http://andaltec.org/documentaci on/pdf/1286263382.pdf

Gong, G., Lu, N., Lu, J. \& Yang, Y. (2012). A single machine scheduling strategy for energy saving in injection molding process. ANTEC 2012.

Gough, A., Gaffney, B. \& Mccann, B. (2006). A Competitiveness Analysis of the Polymer and 
Plastics Industry on the Island of Ireland. InterTradelreland. Plastics Ireland, 88.

Heur, R. Van, \& Verheijen, M. (2009). Power Quality \& Utilization Guide. Plastics Industry, 22. Recuperado de: http://www.leonardoenergy.org/files/root/pdf/Applica tion_Guide_Plastics.pdf

Hill, B. A. \& Figueroa, E. (2007). Administración de los recursos energéticos. Área Metropolitana del Valle de Aburrá. Universidad Nacional de Colombia. Recuperado de. http://www.metropol.gov.co/Pro duccionLimpia/Documents/Uso\% 20eficiente\%20Energia/Administr acion_Recursos_Energeticos.pdf

Huang, Y., \& Yang, G. (2012). The Applied Research of Green Production Technologies Based on the Production in Plastic Molding Factories. Energy Procedia, 14, (1), doi:10.1016/j.egypro.2011.12.88 7

ICIPC. (2013). Instituto de Capacitación e Investigación del Plástico y del Caucho, ICIPC. Gruoplac Colciencias. Recuperado de: http://201.234.78.173:8080/grup lac/jsp/visualiza/visualizagr.jsp?n ro $=00000000003414$

IEA, International Energy Agency. (2013). World energy oulook 2013. World Energy Outlook. Recuperado de: http://www.iea.org/publications/ freepublications/publication/worl d-energy-outlook-2013--executive-summary---spanishversion.html
Ipsom. (2011). Las empresas del sector del plástico pueden ahorrar una media de un $30 \%$ en suministro energético. leetu. Recuperado de:

http://www.leetu.com/2010/09/ 02/las-empresas-del-sector-delplastico-pueden-ahorrar-unamedia-de-un-30-en-suministroenergetico/

Ipsom. (2012). Caso exitoso de ahorro de energía en el proceso de inyección. Recuperado de http://www.ipsom.com/proyecto s/inyeccion/.

Joseph, B. (2003). Energy Efficient Injection Molding Operation. California Edison Company.

KEMA. (2012). Industrial Sectors Market Characterization. Plastic Industry. Oakland, California. Recuperado de:

http://calmac.org/publications/Fi nal_Plastics_Market_Characteriz ation.pdf

Kent, R. (2008). Energy Management in Plastics Processing, (1ra Ed.). Londres, Inglaterra: Plastics Information Direct.

Kent, R. (2008b). Energy management in plastics processing framework for measurement, assessment and prediction. Plastics, Rubber and Composites, 37(2/3/4).

Kent, R. (2009). Introduction to energy management for plastics processors. Plastics Technology. Recuperado de: http://www.ptonline.com/article s/introduction-to-energymanagement-for-plasticsprocessors 
Kent, R. (2011). Energy Management in Plastics Processing. A Signposting Guide by The British Plastics Federation. Recuperado de: www.bpf.co.uk

Kraussmaffei, T. (2010). BluePower Eficiencia por KraussMaffei BluePower. Jornadas de eficiencia energética. Martos, España. Recuperado de: http://andaltec.org/documentaci on/pdf/1286263428.pdf

Ltd, T. T. (2013). TANGRAM TECHNOLOGY Ltd. - Consulting Engineers for Plastics Products. Retrieved from http://www.tangram.co.uk/

Lu, N., Gong, G., Yang, Y. \& Lu, J. (2012). Multi-objective process parameter optimization for energy saving in injection molding process. Journal of Zhejiang University SCIENCE A, 13(5), 382-394. doi:10.1631/jzus.A1100250

McCart, M. \& Kearns, M. (2012). Case Study: Polymer processing research center Queens University. British Plastics Federation. Recuperado de: http://www.bpf.co.uk/Sustainabl e_Manufacturing/Energy_Efficien cy/Polymer_Processing_Research _Centre.aspx

Men Mena, J. \& Estrada, O. (2013). Eficiencia energética en el proceso de extrusión monohusillo con alimentación forzada. ICIPC Colloquium 2013.

Naranjo, A. (2012). Ecoeficiencia en el uso de energía en la industria de plásticos. Tecnología del plástico. Recuperado http://www.plastico.com/tp/secc iones/TP/ES/MAIN/IN/ARTICULO S/doc_89033_HTML.html?idDocu mento $=89033$

Neelis, M., Worrell, E. \& Masanet, E. (2008). Energy Efficiency Improvement and Cost Saving Opportunities for the Petrochemical Industry An Energy Star Guide for Energy and Plant Managers. Ernest Orlando Lawrence Berkeley National Laboratory, 1-132.

Noriega, M. del P. \& Estrada, O. A. (2011). Uso eficiente de la energía en el procesamiento de plásticos. Tecnología del plástico. Recuperado de: http://www.plastico.com/tp/secc iones/TP/ES/MAIN/IN/ARTICULO S/doc_84072_prnIN04.html?idDo cumento $=84072$

Novoa, C. A., Rojas, C. F., Cano, C. C., Rincón, L. M., Sanz, F. S., Caicedo, O. P. y Corredor, A. (2009). Guía metodológica para el uso eficiente de la energía en el sector de plásticos, 52. Recuperado de: http://www.caem.org.co/docum entos/600_GUIA_METODOLOGIC A_PLASTICOS.pdf

Padur, P. \& MacDougall, W. (2011). The Plastics Industry in Germany, (2010). Germany Trade \& Invest 10, 1-17.

Pethybridge, E., Hoi, T. \& Mui, D. T. (2002). Plastic Industry Environmental Review. Hanoi University of technology Institute for environmental science and institute for environmental science and mental science and 
technology. Technology Vietnam Cleaner Production Centre, 1-55.

PlasticsEurope \& Organisations, (2012). Plastics - the Facts 2012 An analysis of European plastics production, demand and waste data for 2011. Recuperado de: www.plasticsconverters.eu

Prias Caicedo, O. F. (2010). Programa de uso racional y eficiente de energía $y$ fuentes no convencionales - PROURE. Bogotá - Colombia. Ministerio de Minas y Energía República de Colombia. Plan de Acción 20102015, 1-150.

RECIPE (2006). Low Energy Plastics Processing. European Best Practice Guide, 80. Recuperado de:

http://www.euRECIPE.com/defau It.asp? Lang $=4 \&$ Page $=21$

RECIPE (2005). European Benchmarking Survey of Energy Consumption and Adoption of Best Practice. Reduced energy consumption in plastics engineering. Recuperado de:

http://www.seai.ie/Your_Busines s/Large_Industry_Energy_Networ k/Workshop_Presentations/Plasti cs_recipe.pdf

René, J., Larrotta, S. \& Rey, O. P. (2000). Situación energética general del sector de los plásticos en Bogotá. Bogotá: Universidad de los Andes.

Santa María, M., Fehr, N.-H. Von Der, Millán, J., Benavides, J., Gracia, O. \& Schutt, E. (2009). El mercado de la energía eléctrica en Colombia: características, evolución e impacto sobre otros sectores. Cuadernos de

Fedesarrollo, No. 30, 62.

Scheihing, P. (2006). Department of Energy?: Save Energy Now Challenges for Energy-Intensive Industries. In SPI Energy Efficiency Workshop. . Industrial Technologies Program Office of Energy Efficiency and Renewable Energy. Chicago, Illinois, 1-23.

SPI \& Energy, U. S. D. of. (2005). Improving Energy Efficiency at U.S. Plastics Manufacturing Plants. Recuperado de: http://www1.eere.energy.gov/m anufacturing/tech_deployment/p dfs/plastics_report.pdf

Suosa M., F., (2014). Eficiencia energética en una industria de inyección de plásticos: problemas e soluciones. (Tesis de maestría). Universidade do Porto. Portugal.

Tanaka, K. (2008). Assessment of energy efficiency performance measures in industry and their application for policy. Energy Policy, 36(8), 2887-2902.

doi:10.1016/j.enpol.2008.03.032

Thiriez, A. \& Gutowski, T. (2006). An Environmental Analysis of Injection Molding. Recuperado de:

http://web.mit.edu/2.810/www/ Thiriez_ISEE_2006.pdf

UNIDO \& MITI (1995). Plastic Forming Industry. In Output of a Seminar on Energy Conservation in Plastic Forming Industry. Recuperado de:

https://www.unido.org/fileadmin /import/userfiles/puffk/plastic.p df 
Villegas, J. \& Naranjo, A. (2013). Eficiencia energética, caso aplicado al proceso de inyección. ICIPC Colloquium 2013. MedellínColombia.

Weissman, A., Gupta, S. K., Ananthanarayanan, A. \& Sriram, R. D. (2010). A systematic methodology for accurate designstage estimation of energy consumption for injection molded parts. Proceedings of the ASME 2010 International Design Engineering Technical Conferences \& Computers and Information in Engineering Conference. 